\title{
Diagnostische Möglichkeiten bei neuromuskulären Krankheiten
}

Malte E. Kornhuber, Stephan Zierz

Klinik und Poliklinik für Neurologie der Martin-Luther-Universität Halle-Wittenberg, Halle/Saale

psychoneuro 2003; 29 (3): 94-103

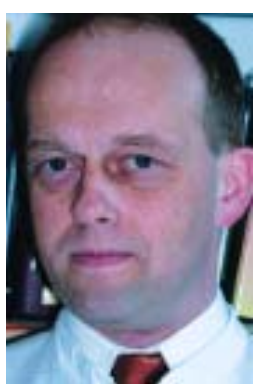

M.E. Kornhuber
Dieser Beitrag gibt einen Überblick über die derzeitigen Möglichkeiten zur Diagnose neuromuskulärer Krankheiten. Der Schwerpunkt liegt dabei auf klinischer und elektrophysiologischer Diagnostik, weil erst auf der Grundlage einer fundierten klinischen Diagnostik gezielt morphologische, biochemische und molekularbiologische Verfahren veranlasst werden können.

$\mathrm{D}$ ie Diagnose der Vielzahl verschiedener neuromuskulärer Krankheiten erfordert eine gründliche klinische und gegebenenfalls elektrophysiologische und klinisch-chemische Untersuchung, um die aufwändigen weiterführenden Untersuchungen, also Muskelbiopsie und evtl. genetische Untersuchung, zum Nutzen des Patienten möglichst gezielt einzusetzen. Dabei ist zu beachten, dass einige neuromuskuläre Krankheiten nicht chronisch progredient verlaufen, sondern sich attackenförmig, episodisch oder belastungsabhängig manifestieren. Im asymptomatischen Intervall zeigen sich dann wenige oder keine klinischen Auffälligkeiten. Dies kann z.B. der Fall sein bei episodischen Paralysen und beim Carnitin-Palmitoyl-Transferase (CPT)Mangel.

\section{Anamnese}

In der Anamnese gilt es Erblichkeit, Manifestationsalter, Verlaufsdynamik und die Charakteristika der
Paresen herauszuarbeiten: Verteilungsmuster, Einfluss von Ruhe, Belastung und Position, von Wärme und Kälte, der Tageszeit, zeitlicher Bezug zu Mahlzeiten, Medikamenten, Situation und ggf. von Infekten. Gibt es Hinweise für weitere Symptome: Myatrophie, (Pseudo)Hypertrophie, Myalgien, myotone Reaktion, Faszikulationen, Krampi bzw. Kontrakturen? Begleitsymptome können sein: Sensibilitätsstörungen, Exanthem, Visusminderung (Katarakt, Retinopathie), autonome, kardiale Symptome etc. Sind bereits in der Kindheit motorische Entwicklungsstörungen fassbar, etwa verzögertes Laufenlernen, schlechtes Abschneiden im Schulsport? Ist in der Vergangenheit Coca-Cola-farbener Urin beobachtet worden als möglicher Hinweis für eine Rhabdomyolyse? Auf anamnestische Hinweise für Begleitkrankheiten (z.B. endokrine Störungen, Neoplasien, Diabetes mellitus) und erbliche Faktoren ist zu achten. Auch weniger naheliegend erscheinende Symptome wie
z.B. Hörminderung sollten erfragt werden, weil diese bei einigen neuromuskulären Erkrankungen überzufällig häufig vorkommen (1).

\section{Körperliche Untersuchung}

Kardinalsymptom vieler neuromuskulärer Krankheiten ist die schlaffe Parese. Muskelhypertonien aufgrund verzögerter Relaxation stellen eine Ausnahme dar (z.B. bei Myotonien). Lähmungen können, müssen aber nicht von Myatrophien (seltener Hypertrophien oder Pseudohypertrophien) und Muskelkonsistenzveränderungen bei Palpation begleitet sein.

Aus dem Verteilungsmuster der Paresen können wertvolle diagnostische Schlüsse gezogen werden auf eine Schädigung im peripheren Nervensystem, auf spinale Muskelatrophien oder auf bestimmte Formen von Myopathien. Lokalisierte Läsionen peripherer Nerven bereiten in der Regel kaum Schwierigkeiten, da es sich um Syndrome mit klar umrissenen motorischen und sensiblen, teils auch autonomen Symptomen handelt. Abgeschwächte Muskeleigenreflexe können sowohl bei Neuropathien, bei spinalen Muskelatrophien und bei Myopathien beobachtet werden. 


\section{Verteilungsmuster der Paresen}

Proximale Myatrophien sind ein häufiges Symptom von Myopathien und auch von spinalen Muskelatrophien, werden aber auch beim myasthenen Syndrom Lambert-Eaton angetroffen. Die Atrophien finden sich im Bereich von Schultergürtel (z.B. Scapula alata, Abb. 1), Rumpfmuskulatur und Gliedergürtel. Dies gilt für hereditäre Gliedergürtelsyndrome und metabolische Myopathien genauso wie für fazio-skapulohumerale Muskeldystrophie, Polymyositis, endokrine und toxische Myopathien sowie für die proximale myotone Myopathie (PROMM, myotone Dystrophie Typ 2).

Neben proximalen Paresen (z.B. Aufrichten-aus-dem-Liegen, aufden-Stuhl-Steigen, die Arme-überdie-Horizontale-Heben) ist auf vermehrte Lendenlordose und Trendelenburgzeichen zu achten. In Ausnahmen können die Symptome ganz auf die Rumpfmuskulatur beschränkt sein wie z.B. bei der axialen Myopathie. Einige Muskelerkrankungen betreffen okuläre oder andere Muskeln im Gesichtsbereich. Beispiele sind die chronisch-progressive externe Ophthalmoplegie, die okulopharyngeale Muskeldystrophie, die fazio-skapulo-humerale Muskeldystrophie und die myotone Dystrophie (Typ 1, CurschmannSteinert). Differenzialdiagnostisch sind kongenitale Aplasiesyndrome von Hirnstammkernen in Erwägung zu ziehen, z.B. Duane-I-Syndrom (Abduzensparese) und MoebiusSyndrom (Abduzens- und Fazialisparese). Ist ein Exophthalmus vorhanden, so ist an eine endokrine Orbitopathie oder eine okuläre Myositis zu denken. Bulbäre Paresen sind häufig bei Myasthenie und motorischen Systemerkrankungen (ALS, Kennedy-Syndrom).

Dysphagie ist ein wichtiges Symptom der okulopharyngealen Muskeldystrophie (Abb. 2), tritt aber auch gelegentlich bei Polymyositis, Einschlusskörper-Myositis und myotoner Dystrophie (Curschmann-Steinert) auf.

Distale Myopathien mit symmetrischer Betonung der Beine bilden eine Gruppe seltener hereditärer Er- krankungen (Abb. 3). Distale Paresen, die auf die Unterarme und Hände beschränkt sind, werden kaum im Rahmen von Myopathien beobachtet. Bei überwiegend asymmetrischen distalen Paresen muss neben der multifokalen motorischen Neuropathie (Immun-Neuropathie) bei Jugendlichen bzw. jungen Erwachsenen auch an das Hirayama-Syndrom gedacht werden. Die distale spinale Muskelatrophie vom Typ Aran-Duchenne betrifft symmetrisch Arme und Beine.

Daneben gibt es Myopathien mit eher asymmetrischen Atrophien und Paresen, die sowohl proximal als auch distal betont sein können. Dazu gehören z.B. die Einschlusskörpermyositis und die Sarkoidose-Myopathie.

Generalisierte Paresen gehen mit jeweils unterschiedlicher Dynamik z.B. auf Intoxikationen (Organophosphate, Botulismus etc.), inflammatorische Polyneuropathien, Myasthenia gravis, Kachexie oder z.B. Vorderhornerkrankungen zurück.

\section{Belastungsabhängige Paresen}

Belastungsabhänge Paresen sind das Kardinalsymptom der Myasthenia gravis und vieler metabolischer Myopathien. In der klinischen Untersuchung ist die Belastungsabhängigkeit gezielt zu untersuchen mit dem Simpson-Test, der Haltezeit von Arm, Bein und Kopf sowie dem Hackengang über die Strecke von einigen Metern. Demgegenüber kann es bei metabolischen Myopathien bei unterschiedlich langer Belastung neben Paresen auch zu Myalgien und schmerzhaften Muskelkontrakturen kommen (s.u.).

\section{- Episodische Paresen}

Episodische Paralysen sind relativ selten. Sie kommen im Rahmen von hereditären Ionenkanalerkrankungen als hyper- oder hypokaliämische Paralysen oder im Rahmen von endokrinen Erkrankungen (vor allem Hyperthyreose) vor.

\section{- Myalgien}

Myalgien treten nur in einem Teil der Fälle mit Polymyositis und mit Dermatomyositis auf. Patienten mit akut bis subakut manifestierendem Verlauf klagen häufiger über Myalgien als Patienten mit chronischem Verlauf. Belastungsabhängige Myalgien sowie Paresen (Belastungsintoleranz) sind wesentliche Symptome der metabolischen Myopathien. Bei den Glykogenosen treten die Symptome rasch nach Beginn der Belastung auf und können sich bei gedrosselt fortgesetzter Belastung bessern („second wind“). Einige der Stoffwechseldefekte im Bereich der Glykolyse führen zusätzlich zu einer hämolytischen Anämie und Hyperurikämie. Bei Fettsäureutilisationsstörungen (z.B. CarnitinPalmitoyl-Transferasemangel) werden Belastungsintoleranz und ggf. Pigmenturie z.T nur nach Ausdauerbelastung, Fasten oder im Rahmen konsumierender Erkrankungen manifest. Über Myalgien in Ruhe und vermehrt nach Belastung wird auch von Individuen mit Myoadenylatdesaminase-Mangel geklagt. Inwiefern die Myalgien durch diese relativ häufige und überwiegend asymptomatische Stoffwechselstörung allein bedingt sind, bleibt zu klären.

Neben einer großen Zahl an Patienten mit muskuloskelettalen Schmerzsyndromen gibt es eine Fülle von Patienten mit Myalgien mit oder ohne umschriebene Druckschmerzhaftigkeiten, für die sich bisher trotz umfangreicher Diagnostik kein eindeutiges organisches Korrelat fassen lässt (z.B. Fibromyalgie).

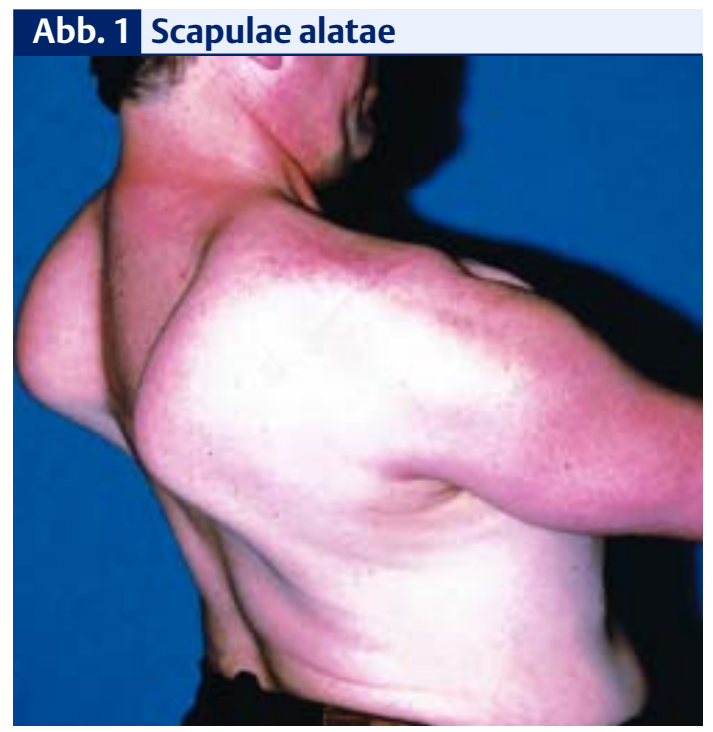

Patient mit Muskeldystrophie vom Gliedergürteltyp. 
Muskelsteifigkeit, Tetanie

Muskelsteifigkeit teils mit Myalgien gibt es jenseits der eigentlichen Myotonien (myotone Reaktion und „warm-up“ unter Belastung nicht immer klinisch fassbar!) auch bei der Polymyalgia rheumatica und

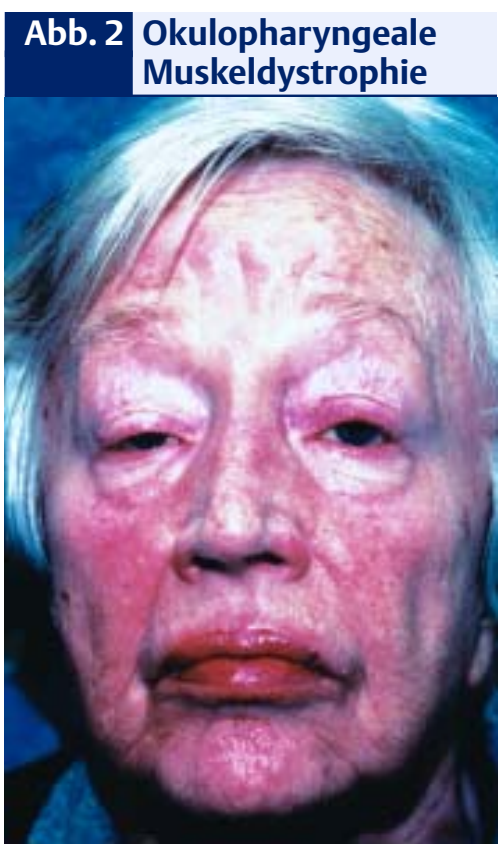

Die Ptose ist bds. bereits mehrfach operativ korrigiert worden. Beachte die Hutchinson-Trias: Bds. Ptose, Anspannung der Musculi frontales, Kopfneigung nach hinten.

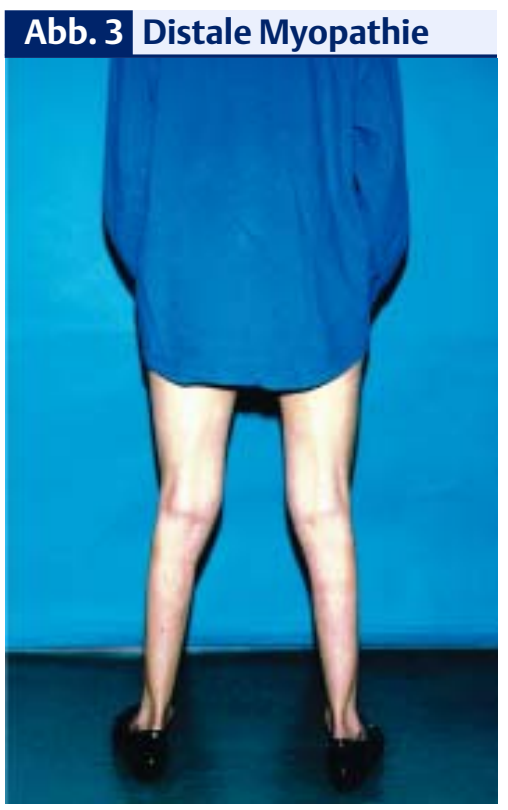

Wadenatrophie bei einer Patientin mit distaler Myopathie vom Typ Markesbery-Griggs/Udd. beim Hoffmann-Syndrom (hypothyreote Myopathie des Erwachsenenalters). Bei Belastung und Kälte zunehmende Muskelsteifigkeit und schmerzhafte Kontrakturen (s.u.) sind wesentliche Symptome der seltenen Brody-Myopathie (Defekt der sarkoplasmatischen KalziumATPase). Ferner treten nicht selten bei zentralen pyramidalen und extrapyramidalen motorischen Syndromen Muskelsteifigkeit und Myalgien auf, die aber in der Regel keine differenzialdiagnostischen Schwierigkeiten bereiten. Auch tetanische Symptome (Karpopedalspasmen, Stridor, Hyperreflexie, Chvostek-Zeichen, Trousseau-Zeichen) bei Elektrolytstörungen bzw. Hyperventilation sind leicht zu erfassen.

\section{- Faszikulationen}

Faszikulationen werden in den terminalen Axonaufzweigungen generiert und müssen keinen Krankheitswert haben. Nicht selten treten sie ohne äußeren Anlass über einen längeren Zeitraum in einzelnen Muskelgruppen auf, ohne dass sich sonstige krankhafte Befunde sichern lassen (benigne Faszikulationen). Gehäuftes Auftreten von Faszikulationen im Kontext mit Krampi und Myalgien wird syndromal als Entität aufgefasst (Faszikulations-KrampusMyalgie-Syndrom), wenn chronische neurogene bzw. motoneuronale Prozesse (z.B. myatrophische Lateralsklerose) unwahrscheinlich sind. Faszikulationen sprechen also eher gegen das Vorliegen einer Myopathie.

Bei ausgeprägtem Faszikulieren ist ferner eine Neuromyotonie (Isaacs-Syndrom) in Erwägung zu ziehen (s.u.). Ähnliche Symptome treten auch bei Überdosierung von Cholinesterasehemmern im Rahmen der Therapie der Myasthenia gravis auf, wobei zu beachten ist, dass es möglicherweise eine pathogenetische Assoziation zwischen Thymom, Myasthenia gravis und Isaacs-Syndrom gibt.

\section{Krampi und}

Muskelkontrakturen

Krampi treten häufig in Kombination mit Faszikulationen auf und sind wie diese Ausdruck der Übererregbarkeit im (terminalen) Axon.
Neben „gutartigen“ Krampi zeigen diese häufig eine chronische neurogene Schädigung an. Neben diesen im EMG durch fortgesetzte Aktivität gekennzeichneten Krampi sind die bei einigen metabolischen Myopathien (z.B. McArdle-Krankheit, Brody-Myopathie) auftretenden, ebenfalls schmerzhaften, transienten Kontrakturen im EMG elektrisch stumm, da es hier aufgrund der metabolischen Störung im Muskel selbst zu einer erschwerten Relaxation des Muskels kommt.

\section{Maligne Hyperthermie}

Die maligne Hyperthermie (MH) stellt eine ernste Narkosekomplikation dar. Die Anlage zur MH kommt unter anderem im Rahmen der Central-Core-Myopathie vor. Die Diagnostik der MH setzt auch heute noch die in-vitro-Exposition von frisch entnommenem Muskelgewebe mit Halothan bzw. Koffein voraus, auch wenn verschiedene Ansätze zur invivo-Diagnostik vorliegen. Auch bei einigen anderen Myopathien ist über $\mathrm{MH}$-ähnliche Phänomene bei Narkose berichtet worden. Dazu gehören z.B. Duchenne/BeckerMuskeldystrophie, Fälle mit hypooder hyperkaliämischer periodischer Paralyse bzw. mit Mitochondriopathie. Gemeinsamer pathophysiologischer Mechanismus dürfte eine Störung der KalziumHomöostase des Muskels mit ungeregelter Aktivierung der kontraktilen Elemente sein.

\section{Gelenkkontrakturen}

Gelenkkontrakturen sind bei einigen seltenen Erkrankungen syndromal mit einer Myopathie vergesellschaftet. Dies ist z.B. bei folgenden kongenitalen Muskeldystrophien der Fall: Emery-DreifussKrankheit, Hauptmann-Thannhauser-Krankheit, Ullrich-Syndrom, Fukuyama-Syndrom. Kontrakturen treten auch im Verlauf verschiedener anderer progredienter Muskeldystrophien auf (z.B. beim Typ Duchenne), wobei frühzeitige orthopädische Maßnahmen hilfreich sind.

\section{Beteiligung anderer Organe}

Gerade bei den hereditären metabolischen Myopathien und Mus- 
keldystrophien ist der Defekt häufig nicht auf den Skelettmuskel beschränkt. Aber auch bei den so genannten Autoimmunerkrankungen können weitere Organe beteiligt sein. Tatsächlich ist $z$. T. die mögliche Herzbeteiligung mit Reizleitungsstörungen oder Herzinsuffizienz Prognose-bestimmend. Dazu zählen z.B. die chronisch-progrediente externe Ophthalmoplegie, die myotone Dystrophie (Curschmann-Steinert), Emery-Dreifuss-Krankheit, Hauptmann-Thannhauser-Krankheit. Es ist wichtig, mit einem Kardiologen zusammenzuarbeiten, der mit der Herzbeteiligung bei diesen Erkrankungen vertraut ist. Z.T. ist die Rate mit plötzlichem Herztod recht hoch, sodass auch die präventive Implantation eines Herzschrittmachers notwendig ist. Augenbeteiligung tritt häufig auf bei Mitochondriopathien (Retinopathie, Optikusatrophie), bei myotonen Dystrophien (Katarakt), seltener bei der fazio-skapulohumeralen Muskeldystrophie (Retinitis zentralis serosa). Bei mitochondrialen Multisystemerkrankungen finden sich gehäuft epileptische Anfälle, Hirninfarkte, Innenohrschwerhörigkeit, Diabetes mellitus.

\section{- Routinelabordiagnostik}

Die Routinelabordiagnostik umfasst die Elektrolyte (Natrium, Kalium, Kalzium), die Blutsenkungsgeschwindigkeit, (alternativ evtl. das C-reaktive Protein), die Creatinkinase (CK) als Muskelenzym, Kreatinin, Alaninaminotransferase (ALT, ALAT, früher: Glutamat-PyruvatTransaminase, GPT) als hepatisches Enzym, TSH basal, und ein Blutbild. Eine „Hyper-CK-Hämie“ erfordert den wiederholten Nachweis erhöhter CK-Werte. Leichte Erhöhungen der CK bis etwa auf das Fünffache des oberen Grenzwertes sieht man auch bei neurogenen Prozessen, insbesondere bei der multifokalen motorischen Neuropathie und bei motorischen Systemerkrankungen. Stärkere Erhöhungen zeigen eher eine primär muskuläre Genese an. Dabei ist anzumerken, dass z.B. sportliche Aktivität insbesondere bei zuvor Untrainierten sowie Allgemeininfekte gelegentlich kurzzeitig mäßige bis deutliche Erhöhungen etwa bis zum 40-fachen des oberen Normwertes nach sich ziehen können. Der Übergang zur Rhabdomyolyse mit z.T. noch weit höheren Werten ist fließend. Adipöse neigen stärker als normalgewichtige Individuen zur belastungsabhängigen $\mathrm{CK}-\mathrm{Er}$ höhung (3). Gelegentlich werden erhöhte CK-Werte durch Makro-CKIsoenzyme vorgetäuscht. Schließlich gibt es auch Individuen mit einer idiopathischen Hyper-CK-Hämie, für die sich unter Ausschöpfung aller diagnostischen Möglichkeiten derzeit keine Ursache finden lässt. Nicht alle neuromuskulären Krankheiten zeigen eine erhöhte CK. Z.B. ist bei metabolischen und insbesondere mitochondrialen sowie endokrinen Myopathien, Myasthenia gravis, Polymyalgia rheumatica nicht regelmäßig mit erhöhten CK-Werten zu rechnen. Je nach Verdachtsdiagnose ist die Labordiagnostik ggf. zu erweitern.

\section{- Elektroneurographie}

Das Elektroneurogramm (ENG) dient vor allem der Untersuchung distaler Extremitätenabschnitte. Dies spielt zur Diagnostik fokaler Neuropathien (z.B. Engpasssyndrome, Leitungsblöcke im Rahmen der multifokalen motorischen Neuropathie etc.) und generalisierter Neuropathien (hereditäre Neuropathien, akutes und chronisches Guillain-Barré-Syndrom) eine große Rolle. Eine Amplituden- bzw. Flächenreduktion des Summenaktionspotenzials entspricht meist einem Verlust an Axonen, kommt aber auch bei Innervationsanomalien, distalem Leitungsblock (zusätzlich Latenzzeitverlängerung!) und bei Myopathien vor. F-Wellen umfassen zusätzlich auch proximale Nervenabschnitte und sind daher wichtig für Erkrankungen mit Beteiligung mehrerer Nervenwurzeln (z.B. GuillainBarré-Syndrom, Cauda-Kompression u.a.). Zunehmend erkennt man die Bedeutung so genannter A-Wellen, also relativ konstanter Reizantworten zwischen Muskel-Antwort und F-Welle. Multiple A-Wellen haben z.B. eine diagnostische Wertigkeit beim Guillain-Barré-Syndrom (2). Ferner hat die F-Wellenableitung eine Bedeutung als Plausibi- litätskontrolle für die übrige Elektroneurographie.

\section{Endplattenbelastungstests}

Eine Objektivierung der Belastungsabhängigkeit einer Parese bei myasthenen Syndromen gelingt mit der Ableitung motorischer Summenaktionspotenziale nach supramaximaler 3/s-Stimulation des entsprechenden Nerven. Das Korrelat ist das reproduzierbare Amplitudenund Flächendekrement der nachfolgenden kleinsten von vier nachfolgenden im Verhältnis zur ersten Reizantwort. Das Dekrement ist nicht spezifisch für die Myasthenia gravis. Es kann z.B. bei Reinnervation, Lambert-Eaton-Syndrom (Abb. 4), Myotonien und Organophosphatintoxikation beobachtet werden. Während die Amplitude des motorischen Summenaktionspotenzials bei der Myasthenia gravis in der Regel normal ist, führt die Antikörper-vermittelte Hemmung der Transmitterfreisetzung beim Lambert-EatonSyndrom dazu, dass bereits die erste Reizantwort eine zu niedrige Amplitude aufweist (Abb. 4). Bei rascher repetitiver Reizung normalisiert sich die Transmitterfreisetzung und es kommt zum diagnostisch beweisenden Amplituden- und Flächeninkrement von mehr als $100 \%$. Tetanische Reizung ist äußerst unangenehm und daher kaum vertretbar. Die repetitive Stimulation erfolgt endogen. Der abgeleitete Muskel wird für $30 \mathrm{~s}$ maximal angespannt. Das Amplitudeninkrement von $>100 \%$ bei nachfolgender elektrischer Stimulation ist beweisend (Abb. 4).

\section{Elektromyographie}

Zur Unterscheidung in neurogene und myogene Prozesse dient jenseits der klinischen Einschätzung in erster Linie das Elektromyogramm (EMG). In der Routineuntersuchung verschafft man sich Klarheit über die Konfiguration von Willkürpotenzialen motorischer Einheiten, deren Rekrutierungsverhalten und über spontan auftretende Phänomene.

Korrelierend zu den morphologischen Veränderungen der motorischen Einheit nehmen Amplitude und Dauer des entsprechenden 
Muskelaktionspotenzials bei neurogenen Prozessen zu, bei myogenen Prozessen ab. Tatsächlich kann es bei lange bestehenden Myopathien teilweise zur Vergrößerung motorischer Einheiten kommen. Umgekehrt können Reinnervationspotenziale bei einer neurogenen Läsion „myopathisch“ anmuten. Dann ist u.a. das unterschiedliche Rekrutierungsverhalten ein sehr wertvolles Unterscheidungskriterium in der klinischen Routine. In einem myopathisch veränderten Muskel müssen im Vergleich zum unversehrten und erst recht im Vergleich zum neurogen veränderten Muskel mehr motorische Einheiten rekrutiert werden, um die gleiche Kraft aufzubauen. Diese „vorzeitige Rekrutierung" ist ein wichtiger Befund bei Myopathien. Bei neurogenen Prozessen wird das Fehlen motorischer Einheiten z.T. durch Vergrößerung verbleibender motorischer Einheiten kompensiert. Bei höhergradigem Axonverlust wird die Kraft aber auch durch vermehrte Entladungsfrequenz der verbleibenden motorischen Einheiten erzeugt (4).

Pathologische Spontanaktivität (PSA; Fibrillationen, positive scharfe Wellen) zeigt eine abnorme Ionenkanalexpression an, die Folge

\section{Abb. 5 Ischämischer Belastungstest am Unterarm}

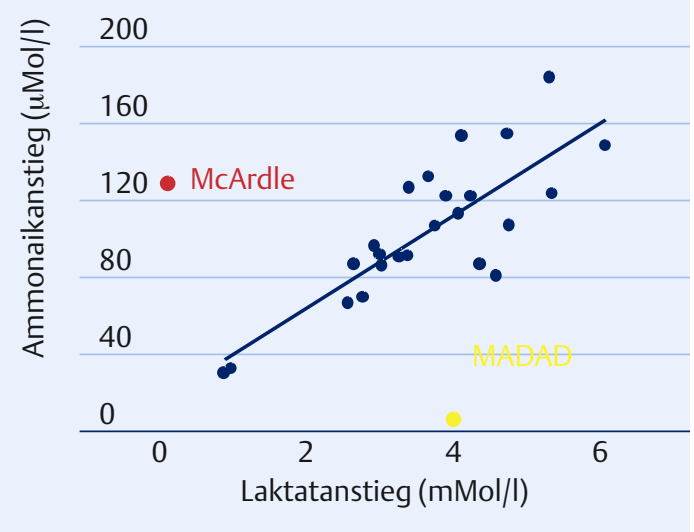

Nach 1-minütiger ischämischer Arbeit steigen Laktat und Ammoniak im lokal entnommenen venösen Blut an. Bei Gesunden (blaue Punkte) korrelieren beide Parameter signifikant. Ein diagnostischer Hinweis auf einen Defekt der Glykolyse oder der Myoadenylatdesaminase ist nur dann gegeben, wenn es selektiv zu einem Anstieg nur eines der Metaboliten kommt.

\section{Abb. 4 Ableitung bei Lambert-Eaton-Syndrom}

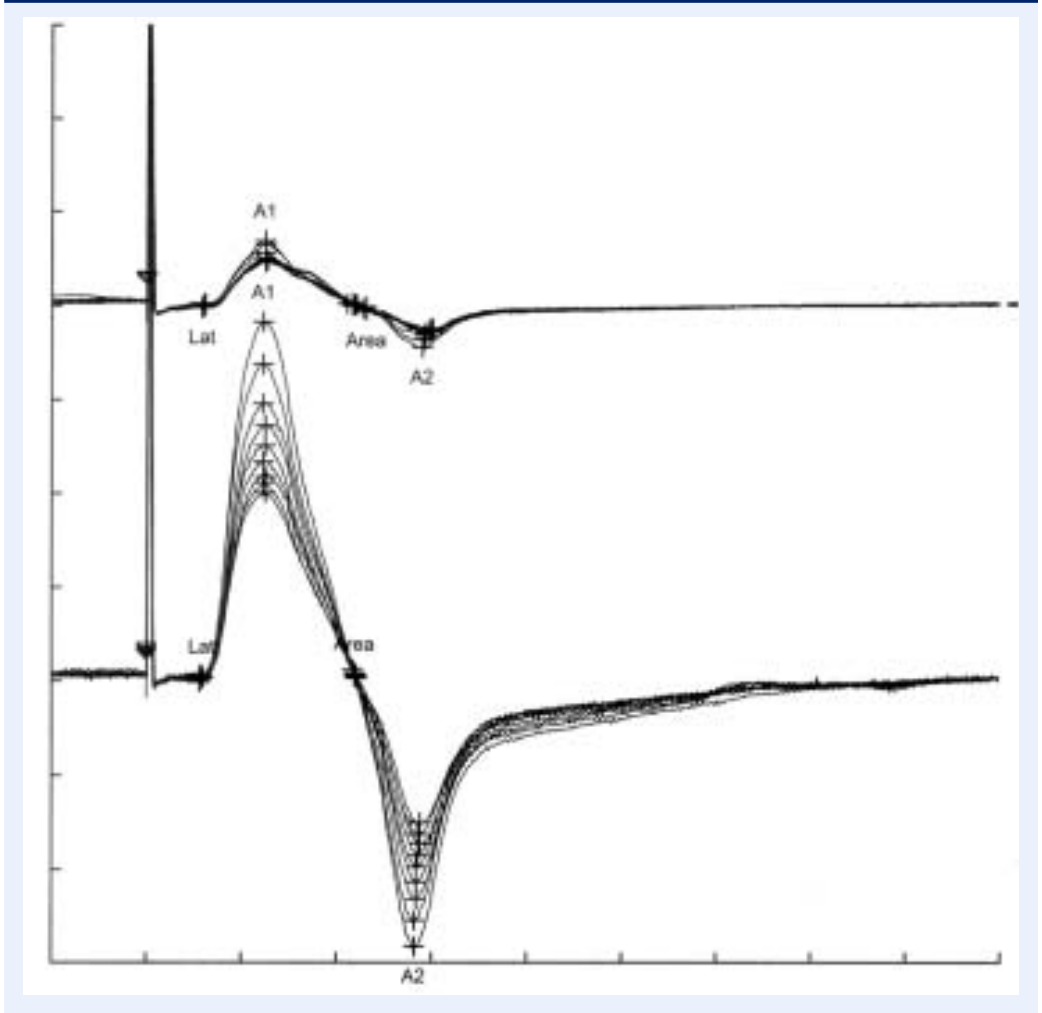

Charakteristischer Befund eines Patienten mit Lambert-Eaton-Syndrom. Ableitung mit Oberflächenelektroden vom M. abduktor digiti minimi links während supramaximaler repetitiver 3/s-Stimulation des N. ulnaris am Handgelenk. Je 9 superponierte Reizantworten. Skaleneinteilung: 1 mV, 5 ms. Die Amplituden der Reizantworten in Ruhe (oben) sind deutlich unter dem Normbereich und zeigen zusätzlich ein signifikantes Dekrement bei nachfolgenden Stimuli. Im Vergleich zu Stimulation in Ruhe (oben) zeigen die Reizantworten nach 30 s maximaler Kleinfingerabduktion (unten) ein signifikantes Inkrement (deutlich über 100\%), wobei bei nachfolgenden Stimuli die Amplitude wiederum zurückgeht.

myogener (z.B. Myositis, Myotonie, metabolische Myopathie - SaureMaltase-Mangel) oder neurogener Erkrankungen sein kann. Bei akuter Denervierung benötigt PSA etwa zwei Wochen zur Ausprägung. Pathologische Spontanaktivität ist ein empfindlicher Befund, mit dem sehr exakt durch Untersuchung mehrerer Muskeln Läsionen im peripheren Nervensystem topisch z.B. einer Nervenwurzel zugeordnet werden können. Charakteristisch für Myotonien sind myotone Serien mit Amplituden- und Frequenzmodulation, die vor allem akustisch erkannt werden, da ihre Geräuschcharakteristik z.T. der eines aufheulenden Motorrades entspricht („Kawasaki-Geräusch“). Hauptcharakteristikum der Neu- romyotonie (Isaacs Syndrom) sind myokyme Entladungen, d.h. ubiquitäre spontane gruppierte Mehrfachentladungen motorischer Einheiten in Form von Dupletten, Tripletten oder Multipletten. Myokyme Entladungen sind nicht spezifisch für die Neuromyotonie. Man findet sie auch bei Tetanien im Rahmen von Elektrolytstörungen, bei der Hyperventilationstetanie, bei fokalen (z.B. Karpaltunnelsyndrom) und generalisierten Neuropathien (z.B. GBS) und bei zentralen Prozessen (multiple Sklerose, spinale Tumore). Nicht immer findet man bei einer Neuromyotonie die charakteristischen und die Erkrankung beweisenden sehr hochfrequenten Entladungsserien, deren Geräuschcharakter entfernt dem eines 
Staubsaugers ähnelt. Es gibt noch eine Reihe anderer spontaner Phänomene im EMG. An dieser Stelle möchten wir z.B. auf die Bedeutung langsamer komplex-repetitiver Entladungen hinweisen, die u.a. bei radiogenen Plexusläsionen nachweisbar sein können.

\section{Biochemische \\ in-vivo-Diagnostik}

Ergeben sich Hinweise für eine metabolische Myopathie, so können klinisch-chemische in-vivo-Muskelbelastungstests wertvolle Befunde liefern. Bei mitochondrialen Erkrankungen ist das Serum-Laktat oft schon in Ruhe erhöht. Die diagnostische Aussage wird dadurch verbessert, wenn es unter standardisierter leichter Fahrradbelastung (30 Watt) zu einem abnorm hohen Laktatanstieg kommt (5).

Demgegenüber lassen sich Hinweise auf einen Defekt der Glykolyse oder der Myoadenylat-Desaminase im Unterarmbelastungstest gewinnen, der üblicherweise unter ischämischen Bedingungen durchgeführt wird (Abb. 5). Kommt es unter Muskelbelastung nicht zu einem Anstieg von Laktat, während Ammoniak deutlich ansteigt, so ist dies ein wichtiger Hinweis auf eine Störung im Glykogen- bzw. Glukosemetabolismus. Beim Mangel an Myoadenylatdesaminase bleibt der Anstieg von Ammoniak unter Belastung aus, während Laktat den erwarteten Anstieg zeigt. Ein fehlender Laktat- und Ammoniakanstieg findet sich u.a. bei ungenügender Mitarbeit (willentlich oder paresebedingt) und ist dann kein Hinweis für eine Glykogenose. Möglicherweise lassen sich ähnliche diagnostische Aussagen auch unter nicht-ischämischen Bedingungen darstellen.

\section{Muskelbiopsie}

Trotz der Fortschritte in der neurogenetischen Diagnostik (s.u.) eröffnet meist erst der histologische, histochemische oder biochemische Befund am Muskelbiopsat die Möglichkeit zur gezielten genetischen Testung und somit die diagnostische Einordnung. Muskelbiopsien werden vom Patienten in aller Regel nicht als besondere Belastung emp- funden. Wichtig ist, dass ein klinisch nicht zu gering und nicht zu stark betroffener Muskel zur Biopsie ausgewählt wird, der nicht in den letzten sechs Wochen einer EMG-Untersuchung unterzogen wurde. Die klinische Untersuchung ist für diese Entscheidung ausreichend. Nur in Ausnahmefällen wird man eine Muskelbildgebung mittels Sonographie oder Magnetresonanztomographie benötigen. Die Muskelbildgebung hat allenfalls einen gewissen Stellenwert zur Verlaufsdiagnostik. Meist werden für die Biopsie der M. bizeps brachii oder der M. vastus lateralis gewählt. Das entnommene Muskelgewebe sollte nicht in Formalin gegeben werden, sondern schonend eingefroren werden, um für histologische, immunhistochemische, histochemische, biochemische und genetische Untersuchungen verfügbar zu sein. Es kommt immer wieder vor, dass Patienten einer Zweitbiopsie unterzogen werden müssen, weil mit formalinfixiertem Muskel keine ausreichenden Aussagen zu erzielen waren. In Einzelfällen sind auch Untersuchungen an nativem Muskelgewebe sinnvoll (z.B. Respirometrie, Isolierung von Mitochondrien etc.), die dann allerdings nur in einem spezialisierten Zentrum erfolgen können.

\section{Neurogenetik}

Genetische Untersuchungen sind nur bei begründetem Verdacht einzusetzen. Auch bei Myopathien mit charakteristischem Phänotyp und bekanntem Genort kann es aufgrund der Vielzahl möglicher und seltener Mutationen sinnvoller sein, zunächst auf Proteinebene (Immunhistochemie, Biochemie, Western Blot aus der Muskelbiopsie) den Defekt nachzuweisen, um dann gezielt die Mutation zu suchen (z.B. bei Dystrophinopathien, Glykogenosen, CPT-II-Mangel). Demgegenüber ist es bei Verdacht auf eine okulopharyngeale Muskeldystrophie, fazio-skapulo-humerale Muskeldystrophie, myotone Dystrophie durchaus sinnvoll, direkt den molekularen Nachweis aus Blutzellen zu versuchen. Bei mitochondrialen Myopathien empfiehlt es sich demgegenüber, die genetischen Untersuchungen an Muskelgewebe vor- zunehmen, da im Blut häufig ein geringerer Heteroplasmiegrad vorliegt und die Diagnose dadurch leichter entgeht. Die Liste der Erkrankungen, die einer neurogenetischen Diagnostik zugänglich sind, wird jedes Jahr länger, sodass im konkreten Fall jeweils neu zu recherchieren ist.

An overview is given about the diagnostic possibilities of neuromuscular diseases. Special attention is devoted to clinical and electrophysiological investigations as they may give rise to further morphological, biochemical or molecular biological procedures.

\section{Key Words}

Neuromuscular disorders - clinical investigation - electroneurography electromyography - biochemical invivo tests - muscle biopsy

\section{Literatur}

1. Deschauer M, Müller T, Schulte-Mattler W, Kornhuber M, Zierz S. Hearing impairment is common in various phenotypes of the mitochondrial DNA A3243G mutation. Arch Neurol 2001; 58: 1885-1888

2. Kornhuber ME, Bischoff C, Mentrup H, Conrad B. Multiple A-waves in Guillain-Barré Syndrome. Muscle Nerve 1999; 22: 394-399

3. Salvadori A, Fanari P, Ruga S, Brunani A, Longhini E. Creatine kinase and creatine kinase-MB isoenzyme during and after exercise testing in normal and obese young people. Chest; 1992: 1687-1689

4. Schulte-Mattler WJ, Georgiadis D, Tietze K, Zierz S. Relation between maximum discharge rates on electromyography and motor unit number estimates. Muscle Nerve 2000; 23: 231-238

5. Zierz S, Meeßen S, Jerusalem F. Lactatund Pyruvatblutspiegel in der Diagnostik mitochondrialer Myopathien. Nervenarzt 1989; 60: 545-548

\section{Korrespondenzadresse:}

M.E. Kornhuber

Klinikum der Medizinischen Fakultät Klinik und Poliklinik für Neurologie

Ernst-Grube-Straße 40

06120 Halle (Saale) 